\title{
Ecoconception des caves : réduction de la consommation d'énergie et intégration paysagère du traitement des effluents de cave avec le dispositif de lit planté de roseaux sur support de zéolithe Zeofito®
}

\author{
J. Rochard ${ }^{1, a}$, E. Marengo ${ }^{2, b}$, D. Marengo ${ }^{3, c}$, J.-L. Mangiacotti ${ }^{4, d}$ et A. Joubert ${ }^{4, e}$ \\ ${ }^{1}$ Vitisplanet, 10200 Bouilly, France \\ 2 Envitech Engineering Sàrl, 1052 Le Mont-sur-Lausanne, Switzerland \\ ${ }^{3}$ Amethyst S.r.l., 12051 Alba, Italy \\ ${ }^{4}$ Serpol, 69333 Lyon, France
}

\begin{abstract}
Résumé. Le traitement aérobie des effluents vinicoles est majoritairement utilisé dans les caves. Au-delà des impératifs de performance d'épuration, avec les orientations de développement durable, le traitement doit intégrer plusieurs orientations : faible consommation d'énergie, limitation des boues, valorisation paysagère et de la biodiversité, limitation des nuisances olfactives et sonores, et éventuellement la réutilisation des effluents traités pour l'irrigation. Les dispositifs classiques de phyto-épuration sur lit de sable, qui permettent de traiter des effluents avec une DCO généralement inférieure à $2 \mathrm{~g} / \mathrm{l}$, imposent le plus souvent un bassin de stockage aéré conséquent, consommateur d'énergie, source éventuelle de nuisance olfactive, visuelle et sonore. Le procédé Zeofito®, qui équipe maintenant une centaine de caves dans différentes régions italiennes, accentue grâce au support de zéolithe, les mécanismes d'adsorption et de biodégradation. Ainsi, il est possible de réaliser un traitement direct ou éventuellement avec un petit bassin de stockage d'égalisation/aération, correspondant à quelques jours de rejet. La réutilisation de l'eau pour le dispositif de refroidissement de la cave ou l'irrigation des vignes est facilitée comparativement aux dispositifs classiques. Parallèlement au principe et aux performances, l'objectif de la communication est de présenter des installations représentatives de la diversité des caves.
\end{abstract}

\begin{abstract}
Aerobic treatment of wine effluents is mainly used in cellars. Beyond the imperatives of wastewater treatment performance, with the orientations of sustainable development, treatment must integrate several orientations: low energy consumption, sludge limitation, landscape and biodiversity enhancement, olfactory and noise pollution limitation, and possibly the reuse of treated effluents for irrigation. Conventional sand-bed phyto-purification systems, which allow effluents to be treated with a COD generally less than $2 \mathrm{~g} / \mathrm{l}$, usually require a large aerated storage tank, which consumes energy and is a potential source of olfactory, visual and noise pollution. The Zeofito ${ }^{\circledR}$ process, which now equips about a hundred cellars in different Italian regions, enhances the adsorption and biodegradation mechanisms thanks to the zeolite support. Thus, it is possible to carry out a direct treatment or possibly with a small equalization/aeration storage tank, corresponding to a few days of discharge. Water reuse for cellar cooling or vine irrigation is easier compared to conventional devices. In addition to the principle and performance, the objective of the communication is to present installations that are representative of the diversity of the cellars.
\end{abstract}

\section{Introduction}

La construction d'un bâtiment viticole, et le choix des équipements associés, supposent une réflexion sur les aspects économiques, qualitatifs et sécuritaires. Audelà de ces aspects fonctionnels, la notion d'écoconception des bâtiments se définit par la prise en compte du développement durable. Comme le spécifie B. PEUPORTIER «l'éco-conception des bâtiments fait

\footnotetext{
a e-mail: rochard.joel@gmail.com

b e-mail: enrico.marengo@envi-tech.ch

c e-mail: dario.marengo@amethyst.it

d e-mail: jeanluc.mangiacotti@serpol.fr

e e-mail: antoine.joubert@serpol.fr
}

appel aux éco-technologies dans le domaine des économies d'énergies et d'eau, des énergies renouvelables (production d'énergie), de la gestion des déchets (matériaux et effluents), des matériaux à moindre impact, tout en incluant les aspects de confort, de santé et d'intégration paysagère».

L'intégration du concept de développement durable au sein de la filière viticole associe, en premier lieu, une adaptation des itinéraires viticoles et œnologiques aux contraintes environnementales, mais également, avec une vision sur le long terme, une prise en compte des aspects énergétiques et de la gestion de l'eau dans la conception globale des caves, destinées à perdurer pendant plusieurs décennies [1]. 


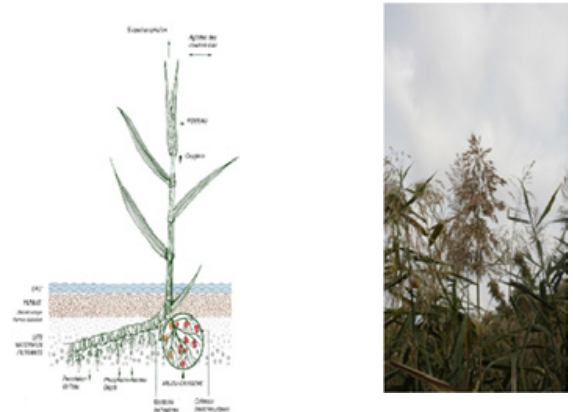

Figure 1. Inflorescence et vue en coupe d'un roseau (Phragmites australis).

Les rejets issus des pressoirs et des caves sont susceptibles de perturber l'équilibre biologique des rivières en particulier pendant la période des vendanges. En effet, les éléments organiques issus des activités vinicoles génèrent, dans un milieu aquatique, le développement de microorganismes qui puisent l'oxygène dissous au détriment de la faune piscicole [2].

Les effluents de cave $(0,5$ à 5 litres/litre de vin) sont de nature organique (DCO de 5 à $30 \mathrm{~g} /$ litre) et sont rejetés majoritairement pendant la période de vendanges (2 à 8 semaines). Le traitement a généralement pour objectif, selon la réglementation locale de réduire la pollution à un niveau de 125 à 300 mg de DCO par litre.

Jusqu'à présent, les procédés de traitement les plus utilisés étaient basés sur des développements technologiques de procédés aérobies et dans une moindre mesure anaérobies. Les recherches actuelles ont pour objectif d'intégrer les orientations de développement durable dans le fonctionnement du dispositif de traitement. Le traitement des effluents doit intégrer différentes orientations : faible consommation d'énergie et limitation des déchets (boues) de plus en plus difficile à gérer par voie agronomique. En complément, et en liaison avec le concept d'éco-onotourisme, une intégration harmonieuse de dispositif peut être envisagée, qui associe à la fois une limitation du nuisances olfactives et sonores, une valorisation paysage et éventuellement de la biodiversité. Bien évidemment, la gestion optimale de l'eau en amont s'impose afin de faciliter le traitement et limiter la raréfaction de la ressource dans de nombreuses régions en liaison avec les changements climatiques.

L'épuration par le sol est un mécanisme utilisé depuis longtemps, notamment la technique d'épandage des effluents. L'épandage des effluents de cave est souvent utilisé sur des terrains agricoles ou de manière plus intensive sur des zones plantées avec des espèces à fort potentiel de développement végétatif (saule, bambou, eucalyptus).

Une autre approche consiste à utiliser le principe d'épuration naturelle des zones humides (phyto-épuration) lié à des plantes dotées d'un fort potentiel racinaire, adaptées à des alternances de conditions sèches et humides $[3,4]$. Certaines de ces plantes (roseaux/Phragmites australis) assurent parallèlement un transfert d'oxygène dans le sol par l'intermédiaire de la tige (Fig. 1).

La phytoépuration a été développée dans les années 1950-1960 suite à la mise en lumière des capacités d'auto-épuration des zones humides. Plusieurs marais artificiels furent par la suite créés dans le but d'épurer

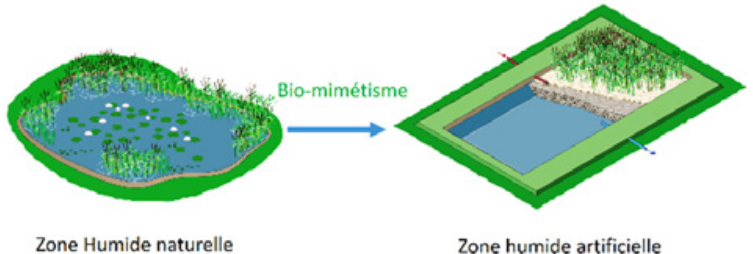

Figure 2. Principe de la phyto-épuration, schéma Zeofito®.

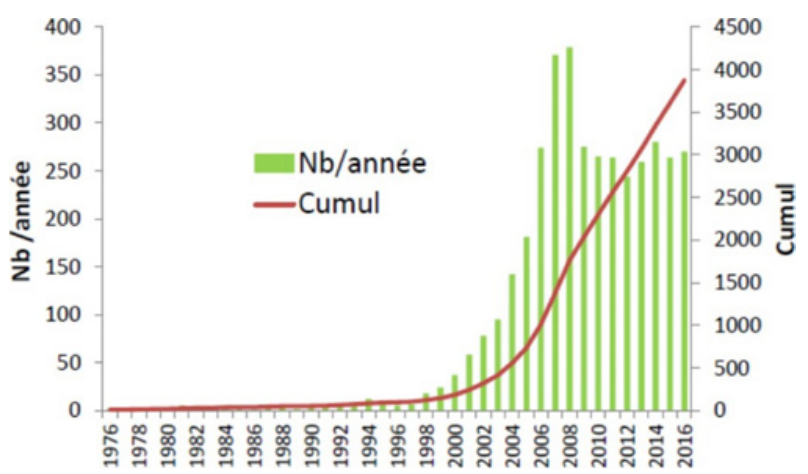

Figure 3. Évolution du nombre de stations d'épuration domestiques par filtres plantés de roseaux en France, source IRSTEA.

les eaux usées (Fig. 2). Le premier marais artificiel fut créé à Othfresen (Allemagne) en 1972. Depuis la fin des années 1990, la phytoépuration est en plein essor en France en Europe. Elle est aujourd'hui une solution reconnue pour le traitement des eaux usées domestiques ou la gestion des boues des stations d'épuration avec l'adaptation progressive à d'autres types effluents agricoles et industriels (Fig. 3). L'application de la phyto-épuration aux effluents de cave a fait l'objet de différentes recherches depuis plusieurs années.

\section{Principe du dispositif de traitement des effluents par lit plante de roseaux}

Le processus reproduit d'une certaine manière le processus naturel d'épuration de l'eau dans les marais où les eaux usées sont naturellement préfiltrées et débarrassées des particules solides, puis subissent des traitements naturels physiques, chimiques et surtout biologiques favorisés par des plantes aquatiques. Celles-ci ont pour effet de dégrader les matières organiques, de transférer vers les feuilles les composés métalliques, de filtrer et de réduire sensiblement les germes pathogènes des eaux usées.

La présence des végétaux induit de façon indirecte un certain nombre de mécanismes favorisant l'épuration : maintien de la structure du massif, apport d'oxygène dans le milieu filtrant et développement de la flore bactérienne [5].

Le massif filtrant est installé dans un bassin d'une profondeur généralement comprise entre $\mathbf{5 0}$ et 70 centimètres et étanchéifié le plus souvent par une géomembrane pour éviter que l'eau non encore traitée ne s'infiltre dans la nappe phréatique.

La présence des végétaux induit de façon indirecte un certain nombre de mécanismes favorisant l'épuration : maintien de la structure du massif, apport d'oxygène dans le milieu filtrant et développement de la flore bactérienne. Parallèlement, les tiges, par leurs oscillations sous l'effet 


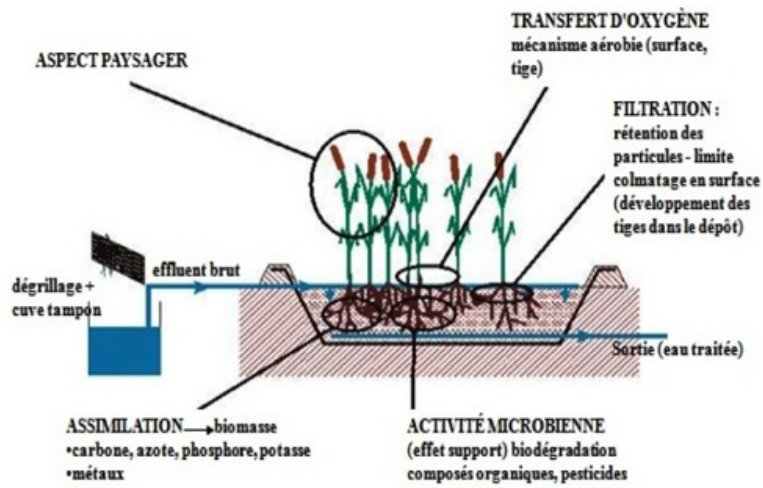

Figure 4. Principe d'un lit planté vertical, d'après J. Rochard.

du vent, maintiennent à leur base un anneau libre qui facilite la circulation hydraulique dans le massif et réduit le colmatage

En complément, les plantes aquatiques, et particulièrement les roseaux (Fig. 4) possèdent un tissu particulier qui permet le transfert d'oxygène depuis les parties aériennes (tiges et feuilles), vers les parties souterraines. Celui-ci est libéré au niveau des jeunes racines dans le film aqueux entourant le « chevelu racinaire $»$. Les bactéries épuratrices présentes à proximité de ces racines sont ainsi alimentées en oxygène.

L'exploitation des capacités épuratoires des massifs filtrants plantés (Fig. 5) peut être mise en œuvre de deux façons :

+ Le lit à flux vertical est un sol artificiel constitué de plusieurs couches de matériaux granulaires superposées, dans lequel se développent les rhizomes. Pour favoriser l'oxygénation du filtre, les eaux à traiter sont injectées de manière séquentielle dans un réseau d'épandage placé à la surface du massif. Le réseau d'alimentation se trouvant en charge à chaque bâchée, l'effluent est réparti de façon régulière, ce qui évite la formation de zones de saturation.

Les effluents percolent par gravité jusqu'à des drains au fond du bassin et sont ainsi évacués dans la partie basse $\mathrm{du}$ système. L'anneau hydraulique autour des roseaux réduit le colmatage, notamment lorsque les effluents sont très chargés en matière en suspension, ce qui permet de traiter des liquides avec des matières en suspension (boues lixiviats, etc.).

Le temps de séjour assez court et l'alimentation séquentielle préviennent la saturation, permettent l'aération du massif et favorisent les phénomènes de dégradation aérobie.

+ Le lit à flux horizontal est un sol artificiel dont les granulométries sont échelonnées en barrières filtrantes selon un vecteur horizontal. Les eaux à traiter, injectées à l'une des extrémités du lit filtrant, pénètrent horizontalement dans la structure puis sont évacuées par drainage à l'autre extrémité.

Parallèlement à leur fonction épuratoire, l'implantation d'un lit planté de roseaux peut s'intégrer dans une démarche paysagère et de biodiversité dans l'environnement de la cave et servir de support à une démarche d'éco-œnotourisme (Fig. 7)

La plupart des dispositifs de lits plantés de roseaux assurent le traitement d'effluents dont la teneur en DCO est proche de 1 gramme par litre, pour atteindre les

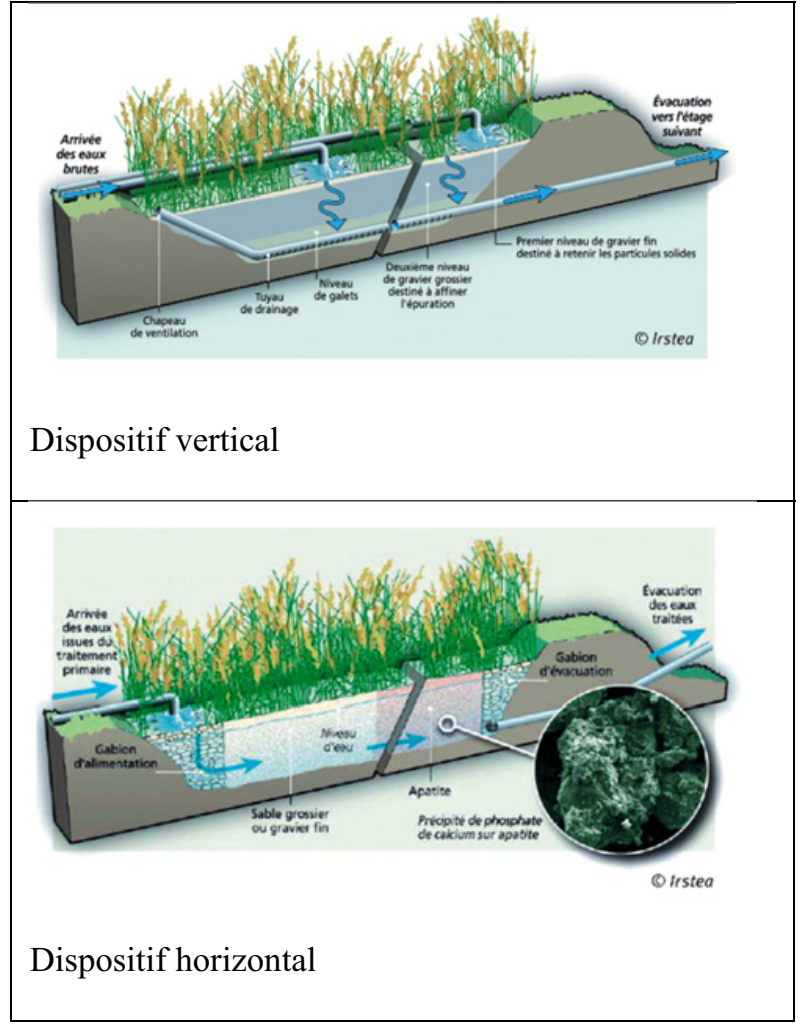

Figure 5. Schéma de principe des lits plantés, source IRSTEA.

normes de rejets, qui varient selon les régions de 125 à 300 milligrammes par litre [4]. Le lit planté est généralement disposé en aval d'un bassin aérobie ou éventuellement d'un dispositif anaérobie, associant selon les cas une épuration de 80 à $95 \%$ pour atteindre un niveau proche de 1 à 1,5 gramme de DCO par litre. Dans ce cas le lit planté, parallèlement au traitement de finition, peut assurer une dégradation des boues du dispositif biologique situé en amont.

\section{Caracteristiques du procede zeofito $\AA$}

L'approche classique de traitement par lit planté en finition impose de maintenir un bassin d'aération important en amont, consommateur d'énergie et source potentielle de nuisance olfactive et visuelle, d'où la recherche de procédés susceptibles de traiter directement les effluents, en associant bien évidemment des mesures en amont $\mathrm{du}$ processus d'élaboration pour limiter la charge et la concentration polluantes.

\section{+ Principe du procédé Zeofito $®$}

Une piste d'optimisation du procédé consiste à utiliser un matériau très adsorbant comparativement au sable ou au gravier des filtres traditionnels. En effet le substrat a plusieurs fonctions: effet support pour les racines: filtration, habitats pour les microorganismes et éventuelle rétention des micropolluants avant leur transfert vers les tiges et les feuilles. Ainsi se combinent notamment des effets d'adsorption, de stabilisation et de précipitation. Afin d'optimiser ce processus, la société italienne Amethyst a eu l'idée d'utiliser la zéolithe, matériau d'origine volcanique dotée d'une microporosité et d'une 


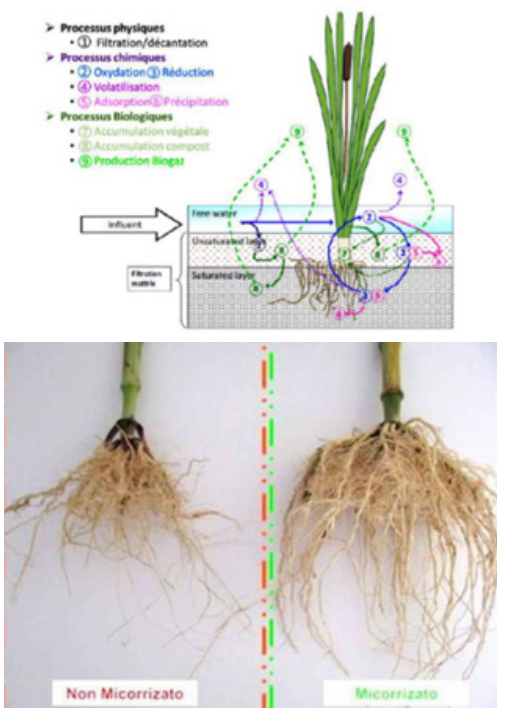

Figure 6. Mécanisme à l'intérieur d'un lit planté Adaptée de F. Chazarenc, 2013 IRSTEA et rizomes de roseaux.

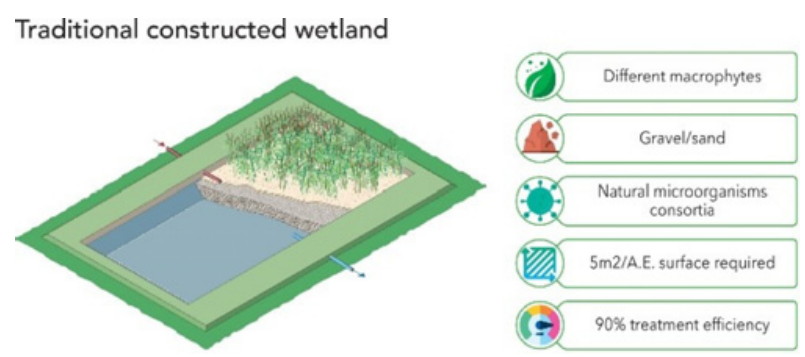

Patented Zeofito ${ }^{\star}$ constructed wetland
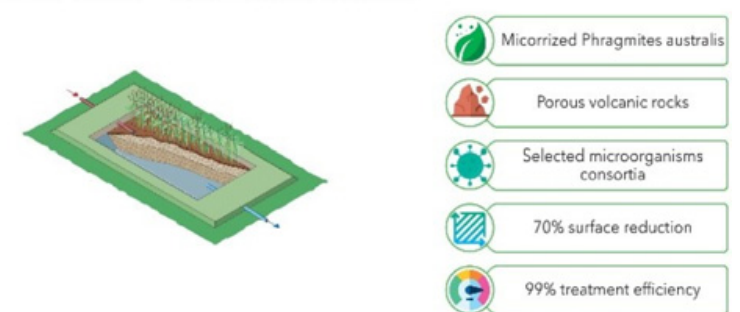

Figure 7. Schéma comparatif d'un lit planté classique sur sable (en haut) et d'un dispositif sur zéolithe (en bas), source Zeofito ${ }^{\circledR}$.

capacité d'adsorption intéressante, tout en associant une très bonne stabilité dans le temps. Cette variante par rapport aux dispositifs classique a donné naissance au procédé Zeofito®.

Une expérimentation a été menée dans une petite cave de Barolo, Podere Ruggeri Corsini, en 2008 (Rochard et al.) en Italie, avec un filtre composé de zéolite en traitement direct sans dispositif aérobie. Les mesures réalisées pendant une campagne de vendange et de vinification ont montré la possibilité de traiter des effluents d'une teneur moyenne de $\mathbf{3}$ grammes de DCO avec une concentration moyenne de l'effluent traité proche de 100 mg/litre (Fig. 8) [6].

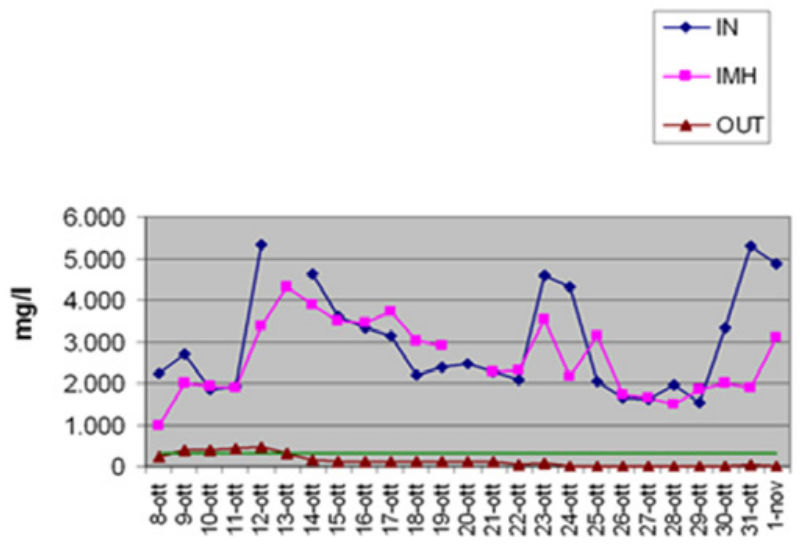

Figure 8. Teneur en DCO des effluents « entrée» après décantation (IMH) et 《sortie 》 du dispositif Zeofito ${ }^{\circledR}$ en traitement direct (sans bassin d'aération préalable), d'après Rochard J.et coll.

\section{+ Rôle de la zéolithe}

En 1756, le minéralogiste suédois Axel Frederik Cronstedt découvre la première zéolithe minérale, baptisée 《 stilbite ». Il reconnaît les zéolithes comme une nouvelle classe de minéraux, constitués d'aluminosilicates hydratés et de terres alcalines.

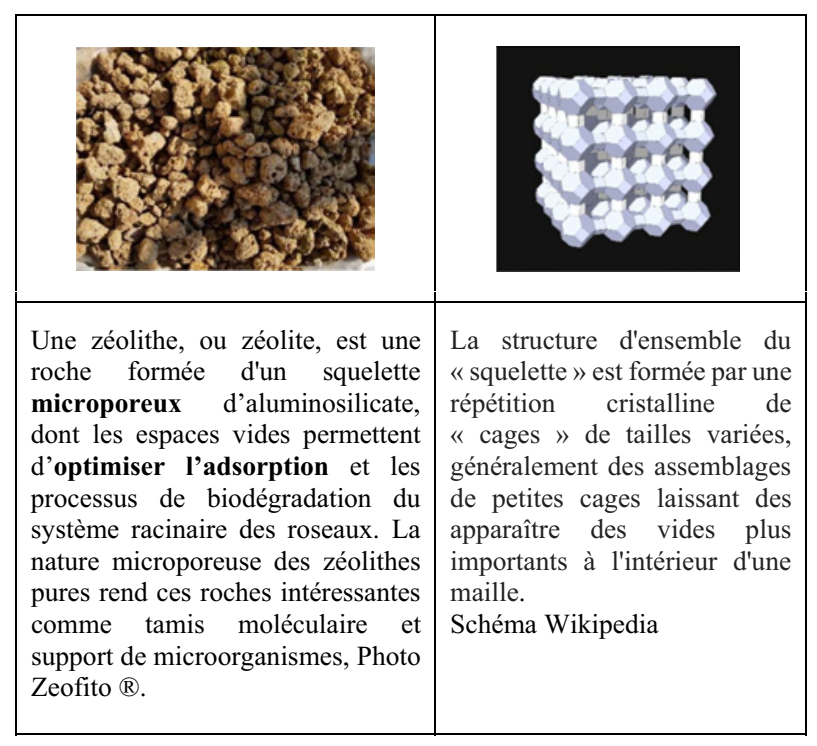

L'observation des propriétés d'expansion en cas de présence prolongée de chaleur (intumescence) conduit Cronstedt a appeler ce minéral « zéolithe 》 du latin zeolithus, du grec $\zeta \dot{\varepsilon} \omega$ (zeô) ou $\zeta \varepsilon \tilde{\iota} \nu$ (zein) : «bouillir» et $\lambda i \theta$ o (lithos) « la pierre»). Une zéolithe, est un cristal formé d'un squelette microporeux d'aluminosilicate, dont les espaces vides connexes sont initialement occupés par des cations et des molécules d'eau. La composition chimique des différentes zéolithes est proche de celle des argiles : ce sont des aluminosilicates plus ou moins hydratés. La différence très importante sur le plan cristallographique est que les argiles présentent une structure feuilletée ou fibreuse tandis que les zéolites présentent une structure tridimensionnelle. Les ions et les molécules d'eau sont mobiles au sein de la structure, ce 


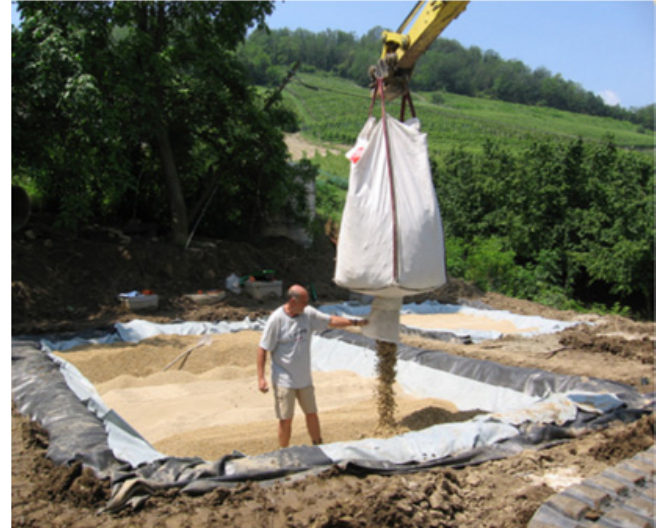

Figure 9. Construction du dispositif Zeofito®, source Amethyst.

qui permet d'une part des échanges ioniques, d'autre part une déshydratation partielle réversible, et la possibilité de remplacer l'eau par une autre phase adsorbée. Le caractère cristallin du squelette implique que les porosités de la structure sont toutes de même taille. Ces porosités peuvent autoriser ou non le passage de molécules (tamis moléculaires).

$\mathrm{Au}$ sein de la rhizosphère, les zéolithes présentes ne saturent jamais, à l'exception du chrome trivalent, mais se régénèrent continuellement grâce à un équilibre renouvelé parmi les cations présents dans les eaux usées et dans la zéolithe. Par exemple, l'ion ammonium $\left(\mathrm{NH}_{4}\right)$ est initialement capturé par échange de cations à partir de la zéolite et ensuite cédé à nouveau par échange de cations, plus lentement et donc à des concentrations inférieures, de sorte que les divers microorganismes tels que le nitrosomonas et nitrobacter peuvent l'oxyder en ion nitrate. L'ion nitrate est en partie utilisé comme élément nutritif par les plantes aquatiques et pour la plupart réduit par les bactéries dénitrifiantes à azote élémentaire $\left(\mathrm{N}_{2}\right)$ et rejeté dans l'atmosphère sous forme de gaz inerte. Même pour les métaux lourds et les radionucléides, le procédé d'épuration suit une méthode similaire à celle décrite ci-dessus. Ces cations, lorsqu'ils sont présents dans les eaux usées, sont d'abord capturés par échange de cations à partir de la zéolite, puis éliminés des effluents et ensuite cédé à nouveau, toujours par échange de cations, mais plus lentement à un niveau de concentration plus faible. Ainsi les racines des plantes peuvent les capturer et les transférer dans la partie aérienne des tiges et des feuilles, où ils s'accumulent.

\section{+ Mise en œuvre}

À la suite de cette expérimentation, de nombreuses caves, notamment en Italie, se sont équipées de ce dispositif en traitement direct, précédé éventuellement d'un petit bassin d'égalisation ou d'aération, avec au préalable un dispositif de dégrillage/filtration et de neutralisation.

Il est intéressant de souligner que ce dispositif, qui s'adapte à chaque contexte de cave (production annuelle de vin, répartition des effluents dans le temps, niveau de pollution, etc.) est mis en œuvre après dégrillage et éventuelle filtration des effluents en traitement direct ou avec un petit bassin d'aération. Il peut également être utilisé en complément d'une station préexistante, dont les performances ne sont pas

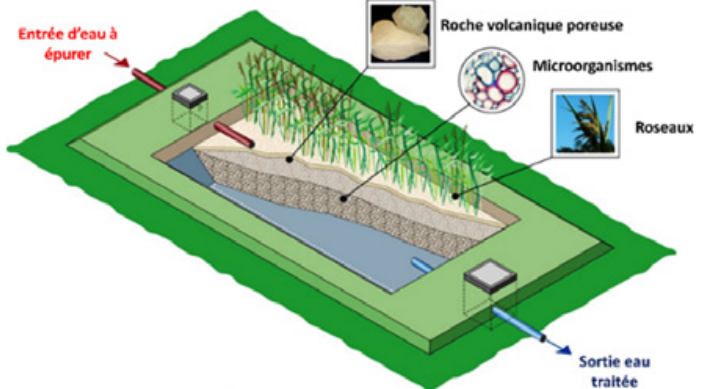

Figure 10. Vue schématique du dispositif de lit planté de roseaux sur zéolite Zeofito®, source Amethyst.

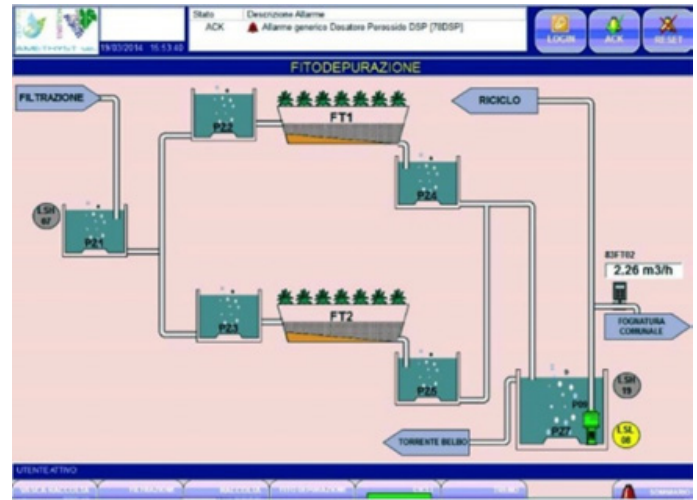

Figure 11. Ecran du dispositif de télésurveillance, source Amethyst.

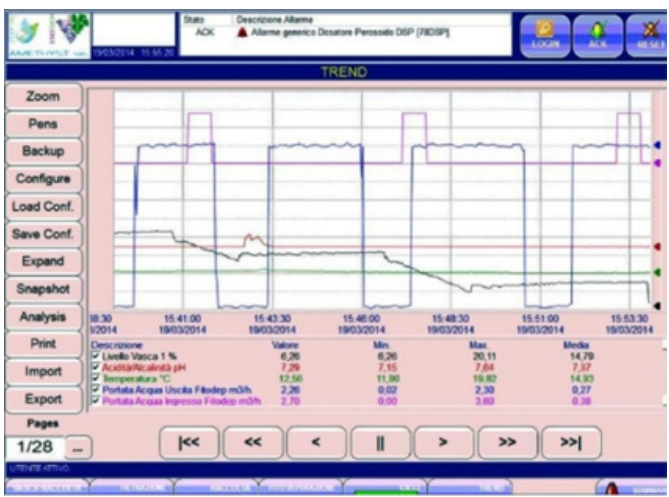

Figure 12. Traçabilité du fonctionnement, source Amethyst.

suffisantes notamment pendant les vendanges, ou encore pour permettre une réutilisation de l'eau (tour de refroidissement ou irrigation). Parallèlement, un filtre planté de roseaux vertical peut être envisagé pour le séchage, la minéralisation des boues, de plus en plus difficiles à gérer par épandage (réglementation, cahiers des charges agricoles), avec une valorisation de la partie végétale par compostage. Un système de télésurveillance avec des systèmes d'alerte peut permettre de suivre à distance le dispositif avec ordinateur ou un smartphone (Figs. 11 et 12).

Des suivis sont réalisés régulièrement afin de de vérifier les performances. À titre d'exemple les Figs. 13, 14 , et 15 , présentent une cinétique des caractéristiques des effluents en amonts et en aval, pour la cave La Battistina avec une teneur élevée en DCO (15 à $\mathbf{2 0} \mathrm{g}$ de $\mathrm{DCO} /$ /itre pendant les vendanges). Cette cave est située dans la région da GAVI dans le Piémont italien. Elle produit 


\section{Entrée/Sortie DCO 1.re Vendange}

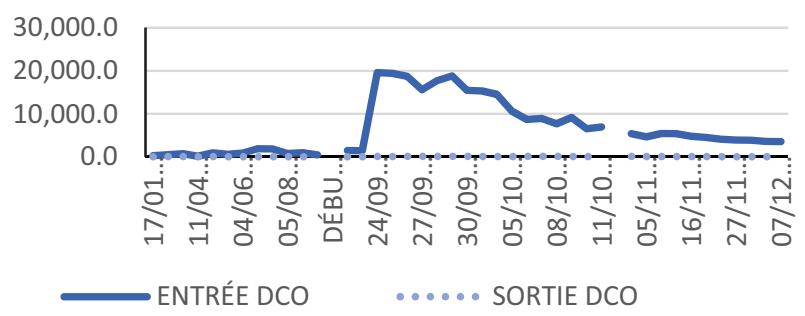

Figure 13. DCO entrée/sortie $[\mathrm{mg} / \mathrm{l}]$ de la cave La Battistina, source Amethyst.

\section{Sortie DBO 1.re Vendange}

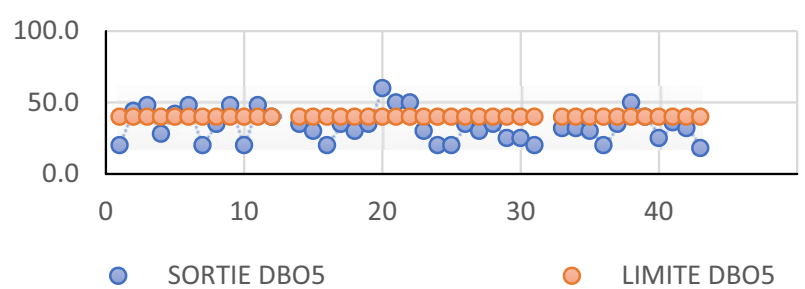

Figure 14. DBO sortie [mg/l] de la cave La Battistina, source Amethyst.

\section{Entrée/Sortie MES 1.re Vendange}

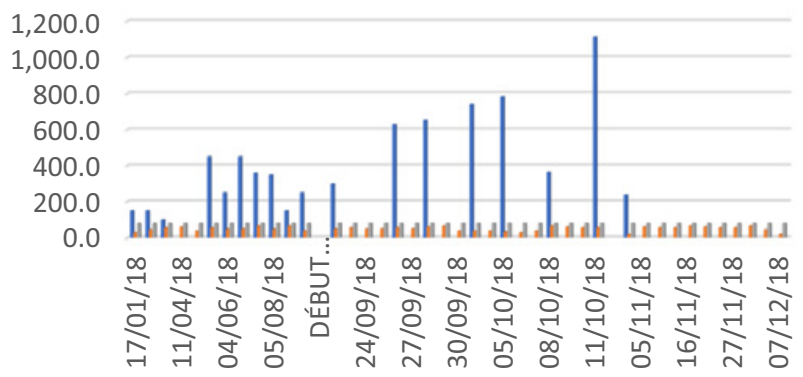

ENTRÉE MES

$$
\text { - SORTIE MES }
$$

- LIMITE MES

Figure 15. MES sortie [mg/l] de la cave La Battistina, source Amethyst.

12.000 hectolitres de vin par an. Le volume d'effluent annuel est d'environ $2.500 \mathrm{~m}^{3}$, dont $950 \mathrm{~m}^{3}$ pendant les vendanges. Le dispositif Zeofito ${ }^{\circledR}$ comporte un bassin d'aération de $80 \mathrm{~m}^{3}$ et 2 lits plantés de zéolithe $\mathbf{d e} 200 \mathbf{~ m}^{2}$. Le fonctionnement de l'installation a démarré au cours des vendanges 2018.

\section{Potentiel de reutilisation de l'eau pour l'irrigation}

Le dispositif Zeofito @ a été testé en traitement complémentaire d'une station d'épuration communale par boues activées (commune de Cossato dans le Piemont en 2007). Le débit optimal du lit planté pour cette station a été établi à $36 \mathrm{~m}^{3}$ par jour pour $50 \mathrm{~m}^{2}$ soit une surface de $0.72 \mathrm{~m}^{2} / \mathrm{m}^{3}$. Concernant l'irrigation ce traitement complémentaire (Fig. 16) a contribué à diminuer de manière très significative les germes pathogènes

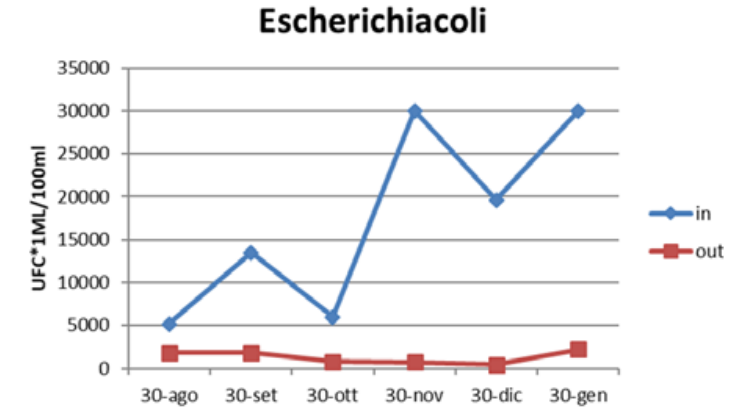

Figure 16. Escherichia coli entrée/sortie. Source CORDAR.

DCO

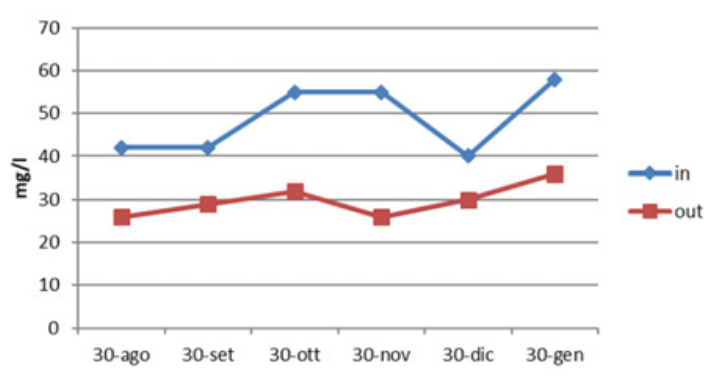

Figure 17. DCO entrée/sortie. Source CORDAR.

(Escherichia coli) ainsi que les matières en suspension (risque de colmatage du dispositif goutte-à-goutte) par rapport à l'effluent en sortie de la station biologique. En complément le dispositif Zeofito a contribué à diminuer sensiblement la DCO (Fig. 17), ainsi que la concentration de tensioactifs.

\section{Exemples}

Quelques exemples, parmi une centaine de caves équipées, témoignent de la diversité des systèmes, adaptée à des contextes différents. Il est utile de rappeler que chaque cave est un cas particulier, ce qui justifie de formaliser précisément les caractéristiques de la cave, établies dans un premier temps à partir d'un questionnaire, portant notamment sur les aspects œnologiques (type de vin, processus d'élaboration, production annuelle, répartition de la pointe d'activité, débits si possible par période et éventuellement pollution domestique complémentaire (habitation, personnel, oenotourisme, restauration, etc.) ainsi que la réglementation locale concernant le rejet vers le milieu naturel ou éventuellement vers la station d'épuration. Ce questionnaire est généralement complété par une ou plusieurs visites sur place, afin d'optimiser la conception et le dimensionnement et éventuellement définir les mesures internes au sein de la cave (économie d'eau, séparation des réseaux, récupération des sous-produits et terre de filtration, etc.).

Pour l'ensemble des exemples, la DCO en sortie imposée par la réglementation est de $160 \mathrm{mg}$, mis à part la cave Fontanafredda, pour laquelle un recyclage de l'eau notamment pour le le bassin d'agrément et le dispositif de refroidissement, a justifié une limite à $15 \mathrm{mg}$ et celle de Nizza qui rejette dans le réseau communal 


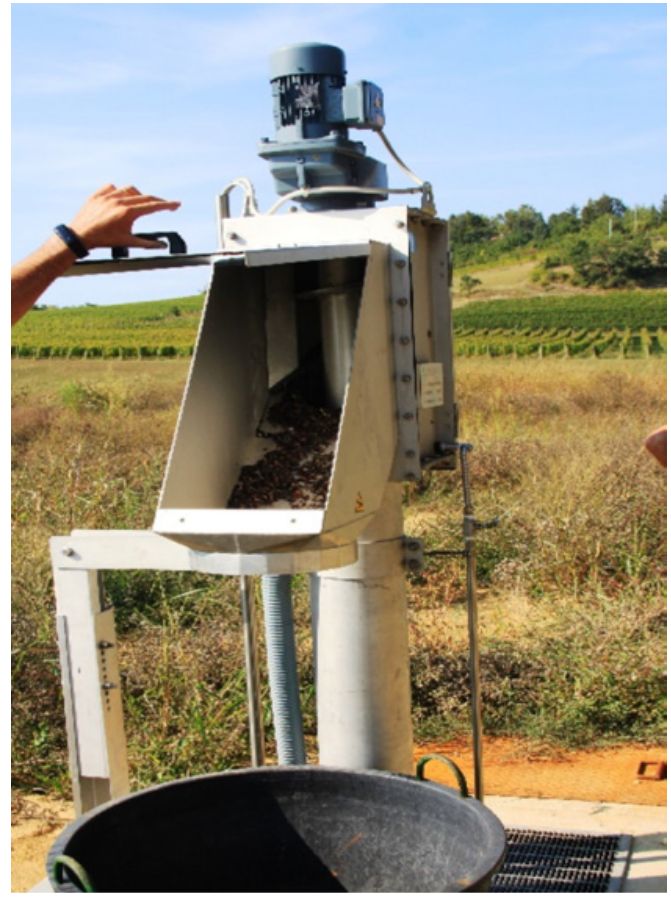

Figure 18. Exemple de dégrillage par vis verticale.

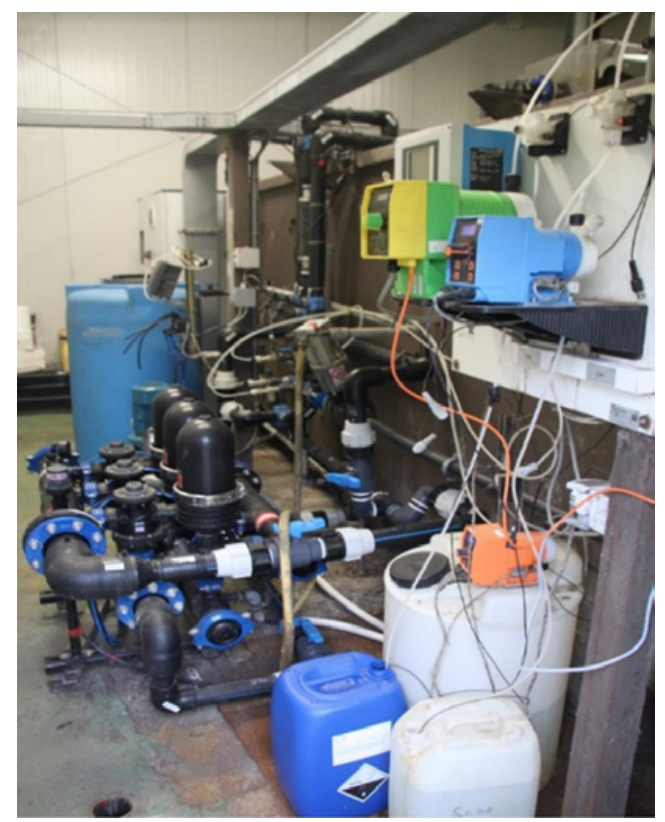

Figure 19. Dispositif de filtration et de régulation de $\mathrm{pH}$, Source Amethyst.

(limite de $1500 \mathrm{mg} / \mathrm{l}$ DCO. Les effluents traités font fait l'objet d'un autocontrôle et d'un suivi complémentaire par les autorités locales. Les valeurs prescrites ont globalement été atteintes mis à part éventuellement un léger dépassement dans la phase de mise en route initiale de la station.

Tous ces exemples disposaient d'un dispositif de dégrillage (Fig. 18) et éventuellement de filtration et de régulation de $\mathbf{p H}$ (Fig. 19).

Généralement les roseaux sont coupés chaque année à la fin de l'hiver et valorisés par compostage.

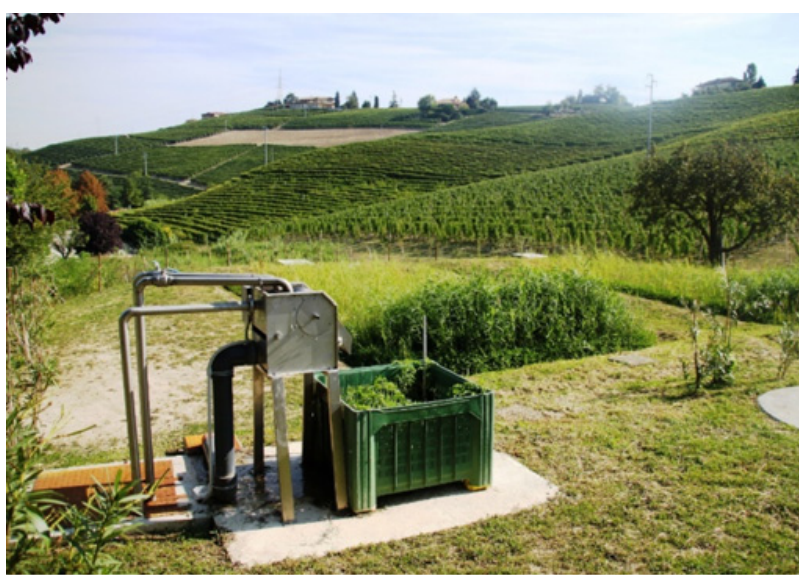

Figure 20. Système Zeofito® de dégrillage et lit planté. Source Amethyst.

+ Cave GAJA, région du Barbaresco en Piémont (Italie).

\begin{tabular}{|l|l|}
\hline Production moyenne de vin/an en $\mathrm{Hl}$ & $5.000,00$ \\
\hline Volume d'effluent moyen/an en $\mathrm{m}^{3}$ & $1.500,00$ \\
\hline Régulation pH & non \\
\hline Cuve tampon & Non \\
\hline Filtration des effluents & non \\
\hline Surface des lits plantés de zéolithe & 2 lits de $80 \mathrm{~m}^{2}$ \\
\hline
\end{tabular}

Le système Zeofito® a été installé en 2007 en traitement direct sans bassin d'aération. Le positionnement proche des habitations et dans une zone classée « patrimoine mondial UNESCO » très visitée, ne permettait pas d'envisager l'installation de bassins aérés ouvert. Aucune mauvaise odeur n'a été décelée dans ce contexte de végétalisation du lit avec les roseaux, associés à un flux horizontal souterrain au travers de la zéolithe (Fig. 20).

+ Cave Banfi, région du Gavi en Piémont (Italie).

\begin{tabular}{|l|l|}
\hline Production moyenne de vin/an en $\mathrm{Hl}$ & $13.000,00$ \\
\hline Volume d'effluent moyen/an en $\mathrm{m}^{3}$ & $1.700,00$ \\
\hline Régulation pH & Oui \\
\hline Cuve tampon & $\begin{array}{l}\text { Non } \\
\text { (complément } \\
\text { d'une } \\
\text { station } \\
\text { boues } \\
\text { activée } \\
\text { existante) }\end{array}$ \\
\hline Filtration des effluents & Oui \\
\hline Surface des lits plantés de zéolithe & $\begin{array}{l}2 \text { lits de } \\
130 \mathrm{~m}^{2}\end{array}$ \\
\hline
\end{tabular}

La cave Banfi disposait initialement d'une station d'épuration par boues activées dont le fonctionnement, notamment pendant les vendanges a été optimisé par un traitement de finition par lit planté de roseaux sur zéolithe Zeofito® (Fig. 21). 


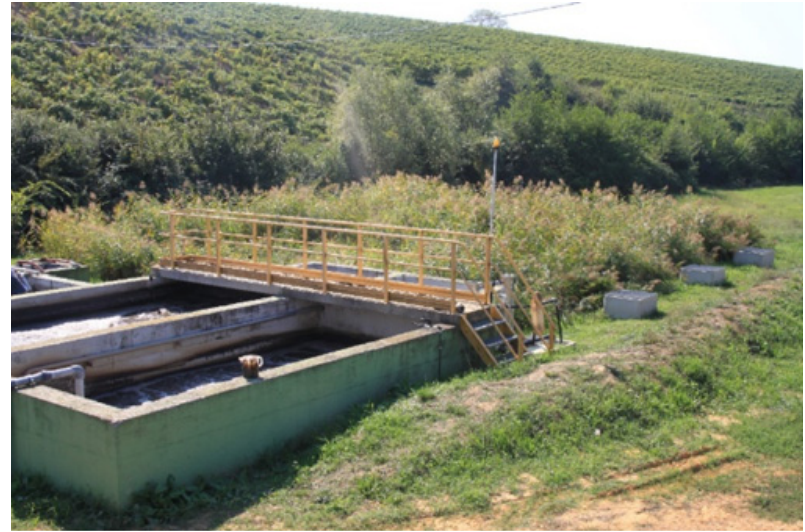

Figure 21. Système Zeofito ${ }^{\circledR}$ de finition en aval de la station «boues activées », cave Banfi, source Amethyst.

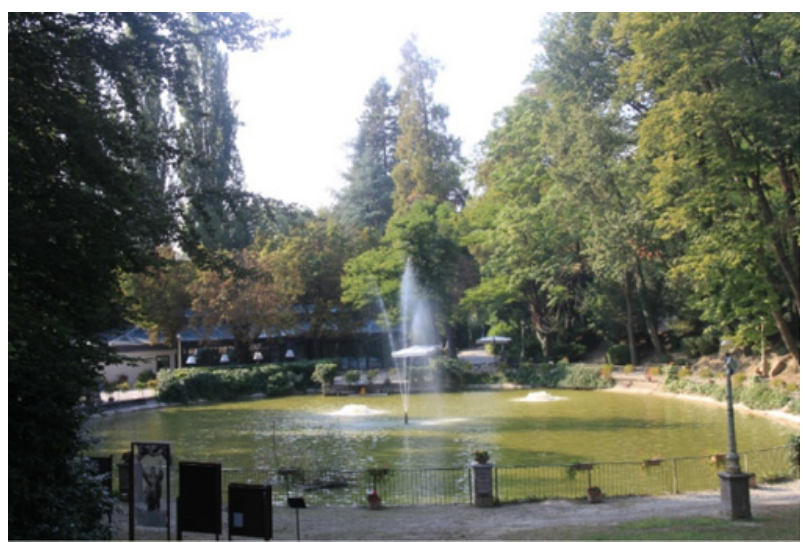

Figure 22. Système d'épuration Zeofito $₫$ avec réutilisation des eaux usées traitées, cave Fontanafredda, source Amethyst.

+ Cave FONTANAFREDDA région du Barolo en Piémont (Italie).

\begin{tabular}{|l|l|}
\hline Production moyenne de vin/an en $\mathrm{Hl}$ & $38.500,00$ \\
\hline Volume d'effluent moyen/an en $\mathrm{m}^{3}$ & $46.000,00$ \\
\hline Régulation pH & Non \\
\hline Cuve tampon & $\begin{array}{l}\text { Non } \\
\text { (complément } \\
\text { d'une } \\
\text { station } \\
\text { physico- } \\
\text { chimique/ } \\
\text { biologique } \\
\text { existante) }\end{array}$ \\
\hline Filtration des effluents & Oui \\
\hline Surface des lits plantés de zéolithe & $\begin{array}{l}2 \text { lits de } \\
120 \mathrm{~m}^{2}\end{array}$ \\
\hline
\end{tabular}

Comme son nom l'indique cette cave a été crée à l'emplacement d'une ancienne source naturelle. Un lit planté sur zéolithe, installé en complément d'une station d'épuration biologique préexistante alimente partiellement un bassin d'agrément, ainsi qu'un dispositif de refroidissent (tours de refroidissement), ce qui a justifié l'exigence de la cave d'une teneur en DCO, en sortie de

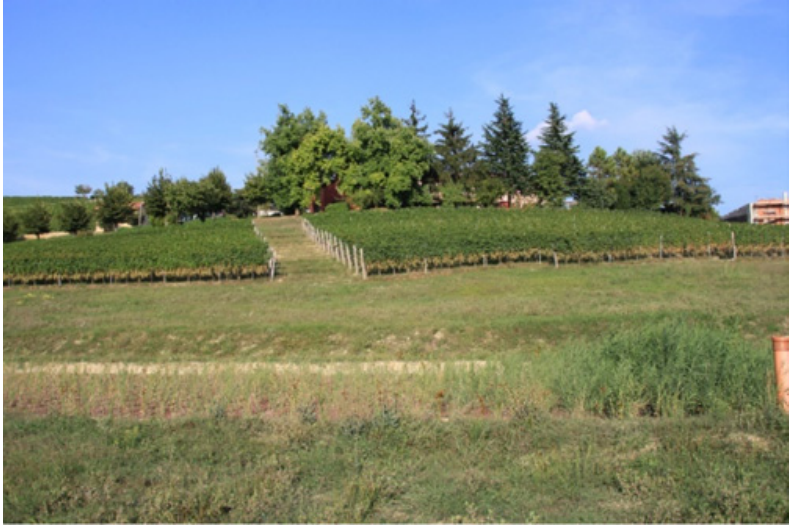

Figure 23. Système Zeofito ${ }^{\circledR}$ de traitement et réutilisation des effluents traités, cave La Battistina, source Amethyst.

traitement, inférieure à $15 \mathrm{mg} / \mathrm{l}$. L'eau du lac est utilisée pour l'irrigation et la lutte contre les incendies (Fig. 22).

+ Cave LA BATTISTINA, région de GAVI en Piémont (Italie).

\begin{tabular}{|l|l|}
\hline Production moyenne de vin/an en $\mathrm{Hl}$ & $12.000,00$ \\
\hline Volume d'effluent moyen/an en $\mathrm{m}^{3}$ & $2.200,00$ \\
\hline Régulation pH & Oui \\
\hline Cuve tampon & $\begin{array}{l}\text { Cuve aération } \\
80 \mathrm{~m}^{3}(4 \text { jours } \\
\text { de temps } \\
\text { de séjour } \\
\text { moyen })\end{array}$ \\
\hline Filtration des effluents & Oui \\
\hline Surface des lits plantés de zéolithe & $\begin{array}{l}2 \text { lits de } \\
200 \mathrm{~m}^{2}\end{array}$ \\
\hline
\end{tabular}

La station a été créée en 2017 avec un dimensionnement adapté une concentration élevée en DCO (environ $15 \mathrm{~g} / \mathrm{l}$ ), liée à une part importante de vinification en blanc, qui a justifié la mise en place en tête d'un bassin d'aération enterré de $80 \mathrm{~m}^{3}$ (Fig. 23).

+ Cave NIZZA, région du Barbera en Piémont (Italie).

\begin{tabular}{|l|l|}
\hline Production moyenne de vin/an en $\mathrm{Hl}$ & $40.000,00$ \\
\hline Volume d'effluent moyen/an en $\mathrm{m}^{3}$ & $4.000,00$ \\
\hline Correction $\mathrm{pH}$ & Oui \\
\hline Cuve tampon & Non \\
\hline Filtration des effluents & Oui \\
\hline Surface des lits plantés de zéolithe & 2 lits de $160 \mathrm{~m}^{2}$ \\
\hline
\end{tabular}

La cave de Nizza est reliée au réseau de la station d'épuration communal, avec un impératif règlementaire inférieur à $1.500,00[\mathrm{mg} / \mathrm{l}]$ pour la DCO. En pratique pendant les vendanges teneur moyenne en DCO à la sortie de la cave est d'environ 11.000,00 [mg/l]. Une zone a été réservée pour un lit planté complémentaire dans une 


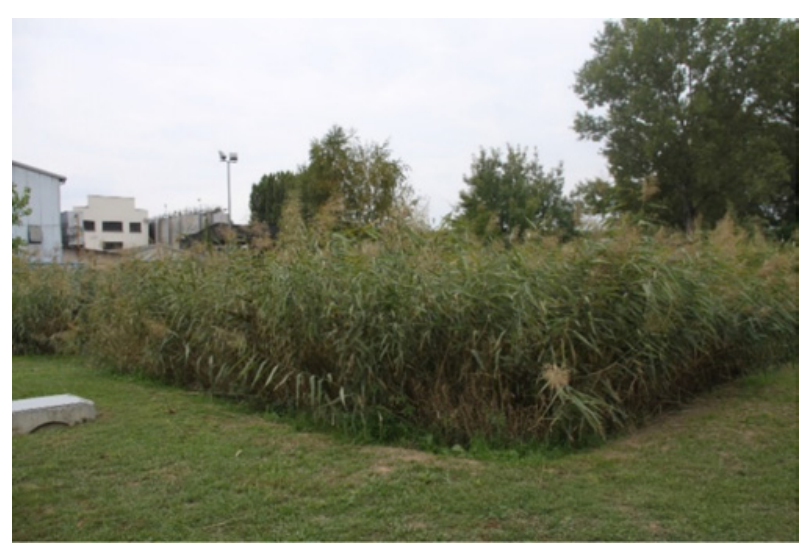

Figure 24. Système Zeofito® de traitement direct, cave Nizza source Amethyst.

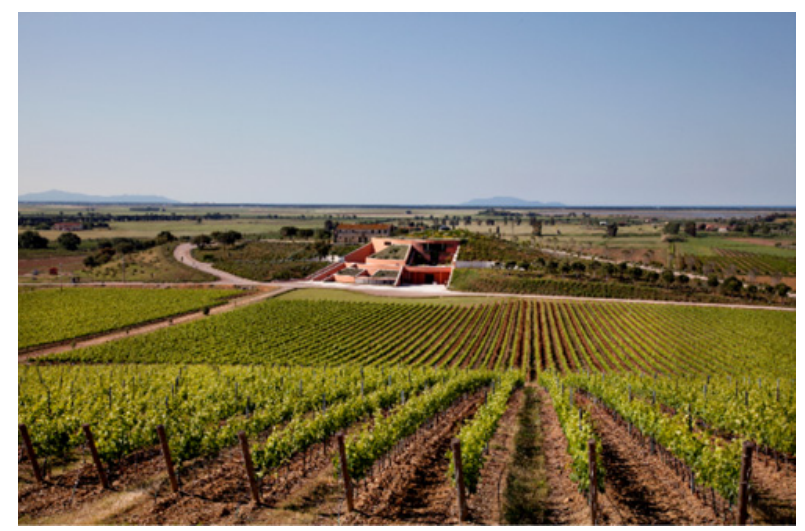

Figure 25. Système Zeofito ${ }^{\circledR}$ de traitement direct, Cave Le Mortelle, Source Amethyst.

perspective future de rejet direct vers la rivière située à proximité (limite de $160 \mathrm{mg} / \mathrm{l} \mathrm{DCO}$ ). Avec l'installation actuelle, pendant la période de vendange, les valeurs de DCO sont généralement inférieures à 500,00 [mg/l]. $\mathrm{Au}$ cours des autres périodes, la teneur de l'effluent en DCO de l'effluent est inférieure à $160 \mathrm{mg} / \mathrm{l}$ ce qui permet potentiellement de rejeter dans le milieu naturel. Ainsi les résultats obtenus sont meilleurs par rapport à ceux envisagés initialement (Fig. 24).

+ Cave Le Mortelle (Groupe Antinori), région de Toscane (Italie).

\begin{tabular}{|l|l|}
\hline Production moyenne de vin/an en $\mathrm{Hl}$ & $6.000,00$ \\
\hline Volume d'effluent moyen/an en $\mathrm{m}^{3}$ & $1.500,00$ \\
\hline Correction pH & Non \\
\hline Cuve tampon & Oui \\
\hline Filtration des effluents & Oui \\
\hline Surface des lits plantés de zéolitheen $\mathrm{m}^{2}$ & $2 * 125\left[\mathrm{~m}^{2}\right]$ \\
\hline
\end{tabular}

La cave italienne Le Mortelle, située à Castiglione della Pescaia en Toscane, a été construite avec pour objectif un très faible impact sur l'environnement et une intégration optimale dans les collines environnantes. La cave a une forme hémisphérique semi-enterrée, cachée en grande

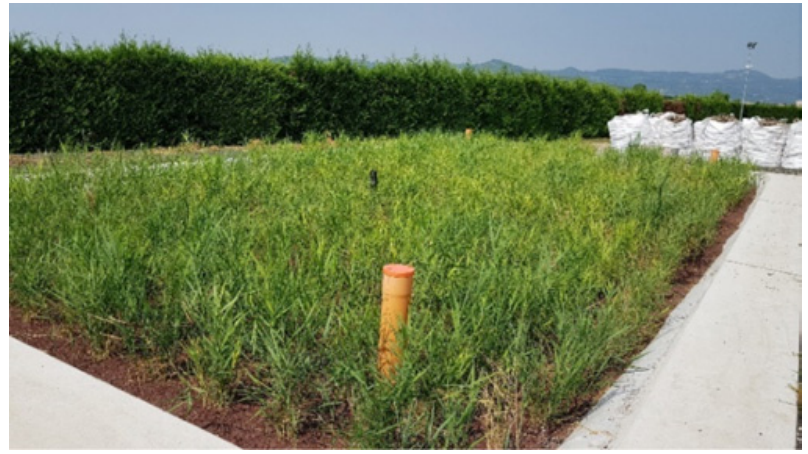

Figure 26. Système Zeofito ${ }^{\circledR}$ de traitement direct après filtration, cave CA' DEL VII, Source Amethyst.

partie dans une colline qui se trouve naturellement dans le domaine. Le choix du dispositif zeofito pour traiter les effluents s'est naturellement inscrit dans le prolongement de l'écoconception de la cave (Fig. 25).

+ Cave CA ' DEL VII région du Prosecco en Veneto (Italie).

\begin{tabular}{|l|l|}
\hline Production moyenne de vin/an en $\mathrm{Hl}$ & $5.000,00$ \\
\hline Volume d'effluent moyen/an en $\mathrm{m}^{3}$ & 500,00 \\
\hline Correction pH & Oui \\
\hline Cuve tampon & Oui \\
\hline Filtration des effluents & Oui \\
\hline Surface des lits plantés de zéolithe & $125 \mathrm{~m}^{2}$ \\
\hline
\end{tabular}

La Cave italienne CA' DEL VII est située à Gambellara en Veneto au cœur de la Région du Prosecco. La station d'épuration est ainsi composée: station de traitement primaire manuelle de correction du $\mathrm{pH}$ et de filtration et station de traitement secondaire de phytoépuration biotechnologique Zeofito®. L'installation est dimensionnée pour rejeter les eaux usées traitées dans le milieu naturel $(\mathrm{DCO}<160[\mathrm{mg} / \mathrm{l}])$, en tenant compte des valeurs d'entrée en septembre et octobre de $\mathrm{DCO}_{M A X}=$ 12.000, 00 [mg/l] (Fig. 26).

\section{Conclusion}

Les lits plantés qui s'inspirent des écosystèmes de milieux humides, s'intègrent dans la diversité des dispositifs de traitement des effluents de cave et de pulvérisation. La conception rustique, la simplicité de gestion, la faible consommation énergétique, la valorisation du paysage et de la biodiversité, sont autant d'arguments qui intéressent les professionnels désireux de développer des démarches durables vis-à-vis des effluents de cave.

Le traitement de finition ou la gestion des boues des dispositifs issus du traitement des effluents domestiques est largement développé.

Afin d'optimiser ce processus, la société italienne Amethyst a eu l'idée d'utiliser un matériau d'origine volcanique la zéolithe, dotée d'une micro-porosité et d'une capacité d'adsorption intéressante tout en 
associant une très bonne stabilité dans le temps, qui a donné naissance au procédé Zeofito®. Maintenant développé dans une centaine de caves, ce procédé, dont les coûts de fonctionnement sont limités, est intéressant dans une perspective de développement durable. La réduction de la consommation d'énergie et le recyclage des effluents traités pour de l'irrigation ou des dispositifs de refroidissement s'intègrent dans une perspective d'adaptation et d'atténuation du changement climatique qui devrait s'amplifier au cours des prochaines décennies.

\section{Références}

[1] J. Rochard, Traité de viticulture et d'œnologie durables, éditions Avenir œnologie (2005)

[2] J. Rochard, F. Jourjon, Y. Racault, Effluents vinicoles, gestion et traitements, éditions Féret (2001)
[3] J. Rochard, Innovation environnementale dans la gestion des effluents de cave : application des lits plantés de roseaux ? 32ème Congrès Mondial de la Vigne et du Vin, Zagreb, Croatie, 28 Juin -3 juillet (2009)

[4] M. Meunier et al., Treatment of Winery Effluent by a Process Combinig an Activated Sludge Reactor with Reed bed Filters, winery waste and ecologic impacts management (2009)

[5] A.R. Muldizi, Winery and Distillery Wastewater Treatment by Constructed Wetland with Shorter Retention Time, 5th international specialized conference on sustainable viticulture: winery waste and ecologic impacts management (2009)

[6] J. Rochard, A. Oldano, D. Marengo, Zeofito ${ }^{\mathrm{TM}}$, The Active Phytopurification System, 5th international specialized conference on sustainable viticulture : winery waste and ecologic impacts management (2009) 